\title{
Dynamical origin of memory and renewal
}

\author{
R. Cakir, ${ }^{1}$ P. Grigolini, ${ }^{1,2,3}$ and A. A. Krokhin ${ }^{1}$ \\ ${ }^{1}$ Center for Nonlinear Science, University of North Texas, P.O. Box 311427, Denton, Texas 76203, USA \\ ${ }^{2}$ Dipartimento di Fisica dell'Università di Pisa and INFM, via Buonarroti 2, 56127 Pisa, Italy \\ ${ }^{3}$ Istituto dei Processi Chimico Fisici del CNR Area della Ricerca di Pisa, Via G. Moruzzi 1, 56124 Pisa, Italy
}

(Received 24 June 2005; revised manuscript received 5 May 2006; published 8 August 2006)

\begin{abstract}
We show that the dynamic approach to fractional Brownian motion (FBM) establishes a link between a non-Poisson renewal process with abrupt jumps resetting to zero the system's memory and correlated dynamic processes, whose individual trajectories keep a nonvanishing memory of their past time evolution. It is well known that the recrossings of the origin by an ordinary one-dimensional diffusion trajectory generates a Lévy (and thus renewal) process of index $\theta=1 / 2$. We prove with theoretical and numerical arguments that this is the special case of a more general condition, insofar as the recrossings produced by the dynamic FBM generates a Lévy process with $0<\theta<1$. This result is extended to produce a satisfactory model for the fluorescent signal of blinking quantum dots.
\end{abstract}

DOI: 10.1103/PhysRevE.74.021108

PACS number(s): 05.40.Fb, 02.50.Ey, 05.60.Cd

\section{INTRODUCTION}

Recent progress in the spectroscopy of nanocrystals and especially of blinking quantum dots (BQD's) [1-3] is generating an increasing interest among theorists, who are challenged by the unusual statistical properties of the BQD spectra. One of them is "aging" [3,4] - a striking property yielding ergodicity breakdown [5] and enforcing reconsideration of the results obtained in the approximation of timeindependent absorption or emission rate [6].

Recent studies of the spectral properties of the BQD raised a question about a dynamic model, which may generate stochastic signals, exhibiting aging (see Ref. [7] for progress in this direction). Stochastic signals or sequences with non-Poisson statistics give rise to the phenomenon of anomalous diffusion [8] - a subject of general interest for different areas of physics. For anomalous diffusion the second moment of the stochastic variable $x$ exhibits scaling with time, $\left\langle x^{2}(t)\right\rangle \propto t^{2 H}$, with $H$ referred to as the scaling exponent. This process is anomalous if $H \neq 1 / 2$. Super diffusion and subdiffusion are realized for $H>1 / 2$ and $H<1 / 2$, respectively. A well-known example of anomalous diffusion is fractional Brownian motion (FBM) [9], yielding a nondecaying correlation function between time $t$ and time $-t$,

$$
\frac{\langle x(-t) x(t)\rangle}{\left\langle x^{2}(t)\right\rangle}=1-2^{2 H-1},
$$

usually interpreted as memory at the level of individual trajectories $[9,10]$. In what follows we propose a dynamic approach to FBM, showing that the FBM memory resides in the velocity $\dot{x}$ but not in the coordinate $x$. As far as the stochastic variable $x$ is concerned, we show that it obeys renewal theory and we argue that the nondecaying corelation function $<x\left(t_{1}\right) x\left(t_{2}\right)>$, evaluated only for positive times, is a manifestation of the FBM nonergodic nature.

On the other hand, the new experimental results on BQD [1-4] are related to a different form of anomalous diffusion $[11,3,6,5,12]$. The emission intermittency of CdSe nanocrystals generates a kind of telegraphic signal, indicating that the system is either in the "light-on" state or in the "light-off" state. This signal has been proved to be a renewal process [3], meaning that each transition resets the system's memory to zero. To establish the relation with anomalous diffusion, the experimental BQD signal is converted into a simplified symbolic sequence. This can be done in two different ways. The first symbolic sequence $\xi_{1}$ associates a transition (regardless of whether it is "on-off" or "off-on") with the values 1 or -1 , according to a fair coin tossing prescription. In the anomalous case of BQD's the time distance between two consecutive jumps is not exponential and the stochastic signal $\xi_{1}(t)$ generates subdiffusion, with aging properties [13]. The second symbolic sequence $\xi_{2}(t)$ results from mapping the BQD fluorescent intensities $I(t)>0$ and $I(t)=0$ to +1 and 0 , respectively. This stochastic signal has been recently studied by Margolin and Barkai [5] and the nonergodic nature of the BQD processes has been demonstrated.

To understand why the stochastic signal $\xi_{1}$ generates subdiffusion when the time distance between two consecutive coin tossing corresponds to the nonergodic BQD condition, we note first of all that ordinary diffusion, with $H=1 / 2$, is derived by a periodic coin tossing procedure, which makes the time distance between the jumps in opposite directions fluctuate according to the Poisson statistics. However, for the optical signal generated by the BQD's, the time intervals $\tau$ between two consecutive transitions (i.e., the time intervals between successive jumps) are distributed according to the inverse power law

$$
\psi(\tau)=(\theta / T)[(\tau / T)+1)]^{-(1+\theta)} .
$$

This means that the time intervals between the jumps are much longer than in the Poisson case; i.e., the diffusion is slower than the ordinary one. The experimental values of $\theta$ for the BQD's lie in the interval $0<\theta<1$; i.e., the distribution, Eq. (2), has an infinite first moment, $\langle\tau\rangle=\infty$. It is evident, therefore, that in the case of diffusion generated by $\xi_{1}$ the random walker waits a much longer time in a single site than in the case of ordinary diffusion (periodically tossing coin procedure), thus generating a slower processsubdiffusion. It is known in fact [14] that the resulting scal- 
ing parameter $H_{r}$ is given by $H_{r}=\theta / 2<1 / 2$. Here the subscript $r$ means that in this case the departure from ordinary diffusion, $H=1 / 2$, is of renewal origin. Therefore, one may expect that the deviation from ordinary diffusion emerging from the BQD signal is of different origin than that from FBM. In the latter case the anomalous behavior is related to Eq. (1), which is erroneously considered to be incompatible with renewal.

It is well known [15] that the distribution of origin recrossings times generated by ordinary diffusion yields $\psi(\tau)$ of Eq. (2) with $\theta=1 / 2$. We notice that this property is the special case of the more general relation between $\theta$ and $H$,

$$
\theta=1-H,
$$

found years ago by Ding and Yang [16]. Therefore, one would expect that this might lead to a model for BQD's that is not restricted to the condition $\theta=1 / 2$. On the basis of the relation of Eq. (3) the parameter $\theta$ would range from 0 to 1 , thereby meeting the observations of some authors. In fact, while Brokmann et al. [3] proposed $\theta=1 / 2$, the authors of Refs. [1,2] observed in the experiment different from $1 / 2$ values for $\theta$, although lying within the interval $0<\theta<1$. Furthermore, the aging properties of the diffusion generated by $\xi_{1}(t)$ [13] and the nonergodic behavior of $\xi_{2}(t)[5,17]$ have been studied in the whole range $0<\theta<1$ and the adoption of the relation of Eq. (3) would yield a model for the full range of nonergodic condition.

We conjecture that the reasons why the researchers in this field missed this opportunity so far, are related to a prejudice concerning FBM. Ding and Yang [16] have derived the important relation of Eq. (3) from the fractal dimensions of a FBM trajectory. Later, the authors of Ref. [18], on the basis of the trajectory memory of FBM, with the agreement of other authors $[19,20]$, argued that the recrossings events are not renewal and that the derivation of Eq. (3) from the renewal assumption is questionable. They also quote their earlier paper [21], where the renewal assumption was made, as one more example of incorrect derivation. Ordinary diffusion is a renewal process, and consequently, also the $x$-axis recrossing is a renewal one, known as a Lévy process with index $\theta=1 / 2[22,17]$. In conclusion, in the current literature there is a tendency to pay attention to the special case of $\theta$ $=1 / 2$ as the only one acceptable to model BQD's.

In this paper we prove that $\theta=1 / 2$ is a singularity in a different sense: This is the only value from the whole interval $[0,1]$ that is incompatible with the plausible conjecture that the fluorescence of BQD's is a complex phenomenon generated by cooperation.

The outline of the article is as follows. In Sec. II we illustrate a model with two variables $x$ and $\xi$, establishing a connection between renewal and cooperation (RC). The variable $\xi$ takes into account memory and cooperation, and the variable $x$ generates renewal properties. In Sec. III we show that in the asymptotic regime $t \rightarrow \infty$ the variable $x$ exhibits the same statistical properties as the coordinate of FBM process. In Sec. IV we support our arguments by means of numerical simulation. Finally, we devote Sec. V to concluding comments.

\section{RC MODEL}

The RC model is is based on the equation

$$
\dot{x}=\xi(t) .
$$

We assign to the stochastic velocity $\xi(t)$ a long-range correlation function $\overline{\xi(t) \xi\left(t^{\prime}\right)}=\xi_{0}^{2} \Phi_{\xi}\left(t-t^{\prime}\right)$. The long-range properties of the variable $\xi(t)$ are determined by cooperation. In what follows we argue that although the velocity is a correlated process, the coordinate $x$ turns out to be a renewal variable.

Let us illustrate first the cooperative and memory properties of the RC model. We derive $\xi(t)$ from the non-Ohmic bath [23]:

$$
\xi(t)=\sum_{i} c_{i}\left[x_{i}(0) \cos \omega_{i} t+v_{i}(0) \omega_{i}^{-1} \sin \omega_{i} t\right] .
$$

Here the initial conditions $x_{i}(0)$ and $v_{i}(0)$ are randomly selected from the canonical distribution $Z^{-1} \exp \left\{-\sum_{i}\left[\omega_{i}^{2} x_{i}^{2}(0)\right.\right.$ $\left.\left.+v_{i}^{2}(0)\right] /(2 T)\right\}$ and the statistical weight of each oscillator is determined by coefficient $c_{i} \propto \omega_{i}^{(\delta+1) / 2}$.

It is easy to see that the model is ergodic. Let us assume that the frequencies $\omega_{i}$ range from $\omega_{0}=0$ to $\omega_{D}$. The frequencies $\omega_{i}$ always can be assumed to be the rational numbers. We divide the interval $\left[0, \omega_{D}\right]$ into $N$ small intervals $(N$ $\gg 1$ ) and introduce

$$
\omega_{i}=\frac{i}{N} \omega_{D}
$$

Let us first evaluate the correlation function of $\xi(t)$ through the time average. Using orthogonality of harmonic functions with rational frequencies

$$
\begin{aligned}
\overline{\cos \left(\omega_{i} t\right) \cos \left[\omega_{j}(t+\tau)\right]} & =\frac{1}{T} \int_{0}^{T} \cos (t) \cos (t+\tau) d t \\
& =\frac{\delta_{i j}}{2} \cos \left(\omega_{i} \tau\right),
\end{aligned}
$$

one obtains

$\overline{\xi(t) \xi(t+\tau)}_{N}=\frac{1}{T} \int_{0}^{T} \xi(t) \xi(t+\tau) d t=\sum_{i=1}^{N} \frac{c_{i}^{2}}{2}\left(x_{i}^{2}+\frac{v_{i}^{2}}{\omega_{i}^{2}}\right) \cos \left(\omega_{i} \tau\right)$.

Here $T$ is the common period for all $N$ oscillators in the bath. Since the frequencies are rational, it is a multiple of $2 \pi$. It is clear that in the thermodynamic limit $N>>1$, the period $T$ approaches infinity.

To prove that this is identical to an ensemble average we subdivide each of the $N$ cells into $M$ smaller cells, $M \gg 1$. The total number of oscillators now is $M N$. The oscillators belonging to the same $i$ th cell have virtually the same frequency $\omega_{i}$ (and, thus, the same weight $c_{i}$ ), but different initial conditions $x_{i(m)}(0)$ and $v_{i(m)}(0)$. Summation over the "internal" index $m$ within the $i$ th cell is equivalent to the averaging over the canonical distribution with temperature $T$, and it gives 


$$
\frac{1}{M} \sum_{m=1}^{M}\left(x_{i(m)}^{2}+\frac{v_{i(m)}^{2}}{\omega_{i}^{2}}\right)=\frac{T}{\omega_{i}^{2}} .
$$

Now the correlation function for the non-Ohmic bath of $M N$ oscillators is written as follows:

$$
\overline{\xi(t) \xi(t+\tau)}_{M N}=M \overline{\xi(t) \xi(t+\tau)}_{N}=M T \sum_{i=1}^{N} \frac{c_{i}^{2}}{\omega_{i}^{2}} \cos \left(\omega_{i} \tau\right)
$$

The averaging over the canonical ensemble eliminates the "off-diagonal" terms, $\left\langle x_{i} x_{j}\right\rangle=\left\langle v_{i} v_{j}\right\rangle=0,\left\langle x_{i} v_{i}\right\rangle=\left\langle x_{i} v_{j}\right\rangle=0$, and yields the same result for the correlation function,

$$
\overline{\xi(t) \xi(t+\tau)}_{N}=\langle\xi(t) \xi(t+\tau)\rangle_{N}=T \sum_{i=1}^{N} \frac{c_{i}^{2}}{\omega_{i}^{2}} \cos \left(\omega_{i} \tau\right),
$$

thus ensuring the ergodicity of the non-Ohmic bath.

It is straightforward to show that the correlation function $\Phi_{\xi}(t)$ has the following asymptotics [24]:

$$
\Phi_{\xi}(t) \equiv\langle\xi(0) \xi(t)\rangle /\left\langle\xi^{2}\right\rangle \propto \operatorname{sign}(1-\delta) / t^{\delta}, \quad t \rightarrow \infty .
$$

The cooperation is controlled by the parameter $\delta$. The coefficients $\left(c_{i} / \omega_{i}\right)^{2}$ of harmonics in Eqs. (10) and (11) are proportional to $\omega_{i}^{\delta-1}$. Thus $\delta=1$ is equivalent to assigning the same statistical weight to all the frequencies. The adoption of either $\delta>1$ or $\delta<1$ is equivalent to establishing a form of cooperative motion, which yields a strong departure from the white noise condition. Equation (12) shows that the nonOhmic condition $\delta \neq 1$ leads either to a positive (for $0<\delta$ $<1$ ) or negative (for $1<\delta<2$ ) power-law tail of the correlation function $[23,24]$. In the latter case a kind of balance between the positive and negative values of the correlation function can be established directly from Eq. (11):

$$
\int_{0}^{\infty} \Phi_{\xi}(t) d t=0
$$

This property of the correlation function is the necessary condition for the realization of subdiffusion. Here we derive it from the differential equation for the dispersion of the stochastic variable $x(t)$,

$$
\left\langle x^{2}(t)\right\rangle=2\langle\xi\rangle^{2} \int_{0}^{t}(t-\tau) \Phi_{\xi}(\tau) d \tau .
$$

Differentiation of the both parts of Eq. (14) gives

$$
\frac{d}{d t}\left\langle x^{2}(t)\right\rangle=2 D(t)
$$

where

$$
D(t) \equiv\left\langle\xi^{2}\right\rangle \int_{0}^{t} \Phi_{\xi}\left(t^{\prime}\right) d t^{\prime}
$$

plays the role of time-dependent diffusion coefficient. By differentiating the both parts of Eq. (15) and using that $\left\langle x^{2}(t)\right\rangle \propto t^{2 H}$, we obtain the following relation between the scaling parameter of anomalous diffusion $H$ and the measure of cooperation, given by the parameter $\delta$ :

$$
H=1-\delta / 2 \text {. }
$$

According to Eq. (12) the integrand in Eq. (16) is negative at $t \rightarrow \infty$ for $\delta>1$. If the negative contribution to the integral exceeded the positive part, the diffusion coefficient (16) would become negative for $t \rightarrow \infty$. This behavior is impossible because it would mean nonphysical regression of the random walker to the origin, $x=0$. In the opposite case of a predominant positive part, the diffusion coefficient would approach a constant positive quantity, thereby producing ordinary diffusion with $H=1 / 2$. This behavior is in conflict with Eq. (17), which gives $H<1 / 2$ for $1<\delta<2$. Thus, the diffusion coefficient (16) must vanish at $t \rightarrow \infty$, giving rise to the condition (13).

Since the exact behavior of the correlation function at short times is not important once the scaling regime in the diffusion is achieved, we interpolate the asymptotics (12) to $t=0$ by a smooth function, obeying condition (13) [25].

Let us now express the scaling condition in a more rigorous form by moving from the single diffusion trajectory $x(t)$ to an ensemble of them, whose time evolution is described by the probability distribution function $p(x, t)$. Thanks to the Gaussian nature of $\xi(t)$, it is straightforward to prove [26] that at $t \rightarrow \infty$ the distribution $p(x, t)$ approaches the canonical form

$$
p(x, t)=\frac{1}{\left(\pi k t^{2 H}\right)^{1 / 2}} \exp \left(-\frac{x^{2}}{k t^{2 H}}\right)
$$

where $k=$ const.

Let us make the critical assumption, which we check numerically in Sec. IV, that in the scaling regime when Eq. (18) becomes valid, the process $x(t)$ is renewal. The trajectory $x(t)$, which at $t=0$ starts from the origin $x=0$, contributes to the probability $p(0, t)$, due to multiple recrossings of the $x$ axis at later times. Introducing the probability density $\psi_{n}(t)$ of $n$ recrossings, with the last one to occur exactly at time $t$, we get, for the density,

$$
p(0, t) \propto 1 / t^{H} \propto \sum_{n=1}^{\infty} \psi_{n}(t) .
$$

The renewal assumption means that the recrossings are statistically independent, thereby ensuring that the Laplace transform of $\psi_{n}$ is equal to $\hat{\psi}^{n}(u)$, with $\hat{\psi}(u)$ being the Laplace transform of $\psi(t)=\psi_{1}(t)$. In the Laplace representation we have, for $u \rightarrow 0$,

$$
\hat{p}(0, u) \propto u^{H-1} \propto \frac{\hat{\psi}(u)}{1-\hat{\psi}(u)} .
$$

For the power-law distribution (2), with $\theta<1$ and $1-\hat{\psi}(u)$ $\propto u^{\theta}$, Eq. (20) leads to the fundamental relation (3). In Sec. IV we shall prove that the renewal property, which we use to rederive the result of Ding and Yang [16], reflects properly the physics of the RC model as well as the FBM. 


\section{RC MODEL AND FBM}

Before confirming the renewal character of the the variable $x$ of the RC model, let us discuss the connection of this variable with FBM. We remind the reader that RiemannLiouville (RL) and Weyl FBM is obtained by replacing the ordinary derivative on the left-hand side of Eq. (4) with the RL and Weyl fractional derivative, respectively [10]. In order to establish a correspondence between the RC model and one of the aforementioned models we study the behavior of the correlation function for stochastic variable $x(t)$ :

$$
\frac{\left\langle x\left(t_{1}\right) x\left(t_{2}\right)\right\rangle}{\left\langle\xi^{2}\right\rangle} \equiv C\left(t_{1}, t_{2}\right) \equiv \int_{0}^{t_{2}} d t_{2} \int_{0}^{t_{1}} d t_{1} \Phi_{\xi}\left(\left|t_{2}-t_{1}\right|\right) .
$$

Changing the variables $u=\left(t_{1}+t_{1}\right) / 2$ and $v=t_{2}-t_{1}$ and using Eq. (14), the correlation function (21) can be expresses through the dispersion as follows:

$$
C\left(t_{1}, t_{2}\right)=\left\langle x^{2}\left(\left|t_{1}\right|\right)\right\rangle+\left\langle x^{2}\left(\left|t_{2}\right|\right)\right\rangle-\left\langle x^{2}\left(\left|t_{1}-t_{2}\right|\right)\right\rangle .
$$

In the scaling regime the dispersion grows according to the relation $\left\langle x^{2}(t)\right\rangle \propto t^{2 H}$; i.e., the correlation function has the following asymptotics at $t_{1} \rightarrow \infty, t_{2} \rightarrow \infty$ and $\left|t_{1}-t_{2}\right| \rightarrow \infty$ :

$$
C\left(t_{1}, t_{2}\right) \propto t_{1}^{2 H}+t_{2}^{2 H}-\left|t_{1}-t_{2}\right|^{2 H} .
$$

We see that in the limit $\left|t_{1}-t_{2}\right| \rightarrow \infty$ the result of ordinary FBM is recovered. The asymptotic behavior in the RL FBM model, which starts at $t=0$, although being quite similar to that of the standard FBM, is not exactly the same [27]. It is worth mentioning that the correlation function (23) is a nonstationary one.

\section{NUMERICAL EXPERIMENT}

To prove that the renewal approach to Eq. (3) is correct, we generate numerically the $x$-axis recrossing events for $H$ $\neq 1 / 2$. Then we examine the resulting signal with the same technique as that applied to the luminescence of the BQD's to analyze their renewal and aging properties [3].

In the numerical simulations we use a discrete time series $t_{n}=n=1,2,3, \ldots$. To generate the random variable $\xi(n)$ it would be appropriate to adopt the noise $\xi(t)$ produced by Eq. (5). However, for this noise to become ergodic and to include the effects of cooperation the number of oscillators must be very large and that creates numerical difficulties. For this reason we use the algorithm proposed in Ref. [28] to generate noise with the correlation function given by Eq. (12). This algorithm is very efficient in generating ergodiccorrelated stochastic signals without computational problems:

$$
\xi(n)=\frac{2}{\pi} \sum_{m=-\infty}^{\infty} Z_{m+n} \int_{0}^{\pi / 2} \sqrt{\phi(y)} \cos (2 m y) d y .
$$

Here the $Z_{n}$ 's are Gaussian random numbers with $\left\langle Z_{n}\right\rangle=0$ and $\left\langle Z_{n}^{2}\right\rangle=\xi_{0}^{2}$ and the function $\phi(y)$ is determined through its Fourier series, $\phi(y)=1+2 \sum_{k=1}^{\infty} \Phi_{\xi}(k) \cos (2 k y)$, where the Fourier coefficient $\Phi_{\xi}(k)$ is the binary correlator of the random ve-

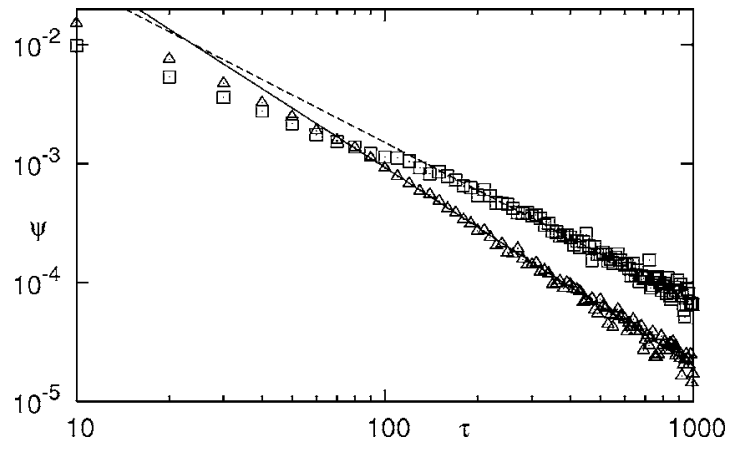

FIG. 1. Numerical results for the waiting time distribution function $\psi(\tau)$ with $H=1 / 3$ (triangles) and $H=2 / 3$ (squares). The former and latter conditions fit the solid line, $2 \tau^{-5 / 3}$, and dashed line, $0.7 \tau^{-4 / 3}$, respectively.

locity $\xi(t)$ calculated at $t=1,2, \ldots$. It is straightforward to check that the algorithm (24) generates a random sequence with binary correlator $\Phi_{\xi}(k)$. In our particular case the exact correlation function is given by Eq. (11) and its asymptotics is given by Eq. (12).

We use the algorithm (24) to generate the series $\xi(n)$ and, from this series, the diffusion trajectory $x(n)=\sum_{k=1}^{n} \xi(k)$ [25]. Then we record the times of recrossing the origin and label by the symbol $\tau_{i}$ the time interval between two consecutive recrossing events. We refer to the times $\tau_{i}$ as waiting times, and we introduce $\psi(\tau)$, being the corresponding distribution density. Two trajectories, one superdiffusional $(\delta=2 / 3, H$ $=2 / 3)$ and one subdiffusional $(\delta=4 / 3, H=1 / 3)$, are generated, and the waiting time distribution functions are plotted in Fig. 1. The slope of the logarithm of the distribution function at $\tau \gg 1$ gives the value of $\theta$ in Eq. (2). In both cases excellent agreement with Eq. (3) is observed.

This, however, is not enough to guarantee the renewal character of the $x(t)$ recrossings of the origin. To afford the proof we did two more numerical simulations. In the first one the normalized correlation function of the waiting times,

$$
\nu_{i k}=\frac{\overline{\left(\tau_{i}-\bar{\tau}\right)\left(\tau_{k}-\bar{\tau}\right)}}{\left(\overline{\tau^{2}}-\bar{\tau}^{2}\right)},
$$

is calculated. It is clear from Fig. 2 that the waiting times are $\delta$ correlated, $\nu_{i k}=\delta_{i k}$; i.e., they are statistically independent. Since $\delta<2$, the first and higher moments of the distribution (2) diverge for a sequence of infinite length. In the numerical calculations the length of the sequence was finite, thereby yielding a finite value for $\bar{\tau}$ and $\overline{\tau^{2}}$, but long enough ( $n$

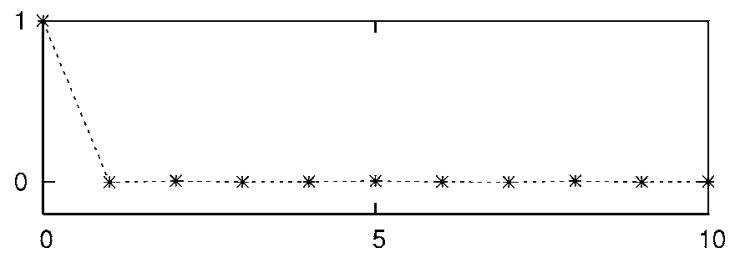

FIG. 2. Normalized correlation function $\nu_{i k}$ given by Eq. (25). It drops to zero after the first step, revealing no correlations ( $H$ $=1 / 3)$. 


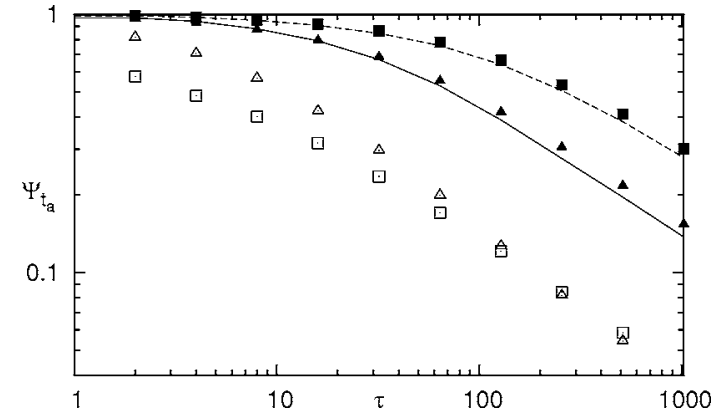

FIG. 3. Aging of the waiting time distribution function for $H$ $=1 / 3$ (triangles) and $H=2 / 3$ (squares). Survival probabilities $\Psi_{t_{a}}$ are plotted for $t_{a}=0$ (open symbols) and for $t_{a}=100$ (solid symbols). Solid and dashed lines refer to the prediction of Eq. (26) for $H$ $=1 / 3$ and $H=2 / 3$, respectively.

$\approx 10^{5}$ ) to provide the convergence on the numerical procedure.

The second simulation aims at confirming the renewal nature of the time sequence $\left\{\tau_{i}\right\}$ by means of the numerical experiment proposed in Ref. [4]. We evaluate the waiting time before the occurrence of the next recrossing event by setting the observation time at a distance $t_{a}$ from each and every previous recorded crossing event. The waiting time distribution function $\psi(\tau)$ shown in Fig. 1 corresponds to $t_{a}$ $=0$. In the nonexponential case the probability of occurrence of the first recurrence, evaluated at $t_{a}>0$ (labeled as $\psi_{t_{a}}$ ), is different from that obtained by setting $t_{a}=0$ and the relaxation of the corresponding survival probability $\Psi_{t_{a}}(\tau)$ $=\int_{\tau}^{\infty} \psi_{t_{a}}(t) d t$ becomes slower and slower with increasing $t_{a}$. That is why this effect is called aging. Although an exact formula for $\psi_{t_{a}}(\tau)$ was derived in Ref. [29], here we use approximation

$$
\psi_{t_{a}}(\tau)=\frac{\int_{0}^{t_{a}} \psi(\tau+t) d t}{t_{a}+\int_{0}^{t_{a}}\left(t-t_{a}\right) \psi(t) d t}
$$

proposed in Refs. [11,30]. Equation (26) gives very accurate approximation for the distribution of the aged waiting times; its accuracy is sufficient for the analysis of the numerical data. It is convenient to illustrate the two results using $\Psi_{t_{a}}(\tau)$ instead of $\psi_{t_{a}}(\tau)$ [31]. In Fig. 3 we plot $\Psi(\tau) \equiv \Psi_{t_{a}=0}(\tau)$ and $\Psi_{t_{a}}(t)$, resulting from the direct numerical evaluation of $\psi(\tau)$ and $\psi_{t_{a}}(\tau)$, together with the predictions of Eq. (26). For both values of $H$ considered, good agreement is observed, thus confirming the renewal aging nature of the waiting time distribution function and, consequently, the renewal character of the recrossing events. For large values of $\tau$ a noticeable difference between numerical and analytical results appears. This is due to insufficient statistical data for $\tau>300$. For the same reason the fluctuations of the random variable $\tau$ in Fig. 1 become stronger in this region.

There may be different types of aging. The aging emerging from the renewal character of a nonexponential process

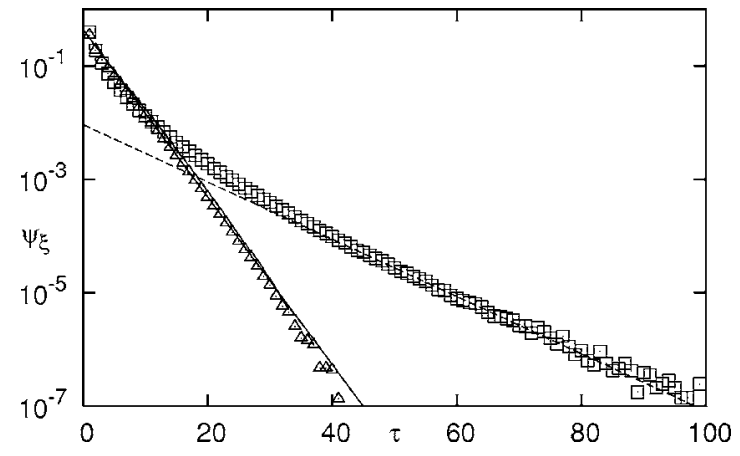

FIG. 4. Waiting time distribution $\psi_{\xi}(\tau)$. The sojourn times $\tau$ refers to the states $\xi(t)>0$ and $\xi(t)<0$. The results for $H=1 / 3$ (triangles) are fitted by the exponential function $0.4 \exp (-0.3 x)$ (solid line), and the results for $H=2 / 3$ (squares) are fitted by $0.01 \exp (-0.1 x)$ (dashed line).

yields Eq. (26). If for a dynamic process the aging fits Eq. (26) at different values of $t_{a}$, this is strong evidence that this process is renewal [4]. We indeed obtained a good fitting (not shown here) to Eq. (26) for some values of $t_{a}$ different from 100.

The RC model of Eq. (4) converts the long-memory tail of Eq. (12) into the non-Poisson renewal properties of $x(t)$. What about the recrossings for the variable $\xi(t)$ ? Figure 4 shows the numerical results for the probability distribution of the corresponding waiting times $\psi_{\xi}(\tau)$, generated by the origin $(\xi=0)$ recrossing times of $\xi(t)$. Both cases considered, $H=1 / 3$ and $H=2 / 3$, generate exponential distributions of the waiting times. This seems to conflict with the long tails of Eq. (12) and with the alleged memory of $\xi(t)$. Actually, there is no conflict, insofar as this is not a renewal process, as confirmed by the extended correlation shown in Fig. 5. This is not surprising since the correlation function of the signal $\operatorname{sign} \xi(t)$ has the same asymptotic properties as $\Phi_{\xi}(t)$. Numerical simulation, not shown here, confirms this expected property.

\section{CONCLUSIONS}

The dynamical model (4) of FBM explains why the renewal character of the recrossing events is compatible with memory and cooperation - the necessary attributes of a complex model for BQD's. In this model the memory and coop-

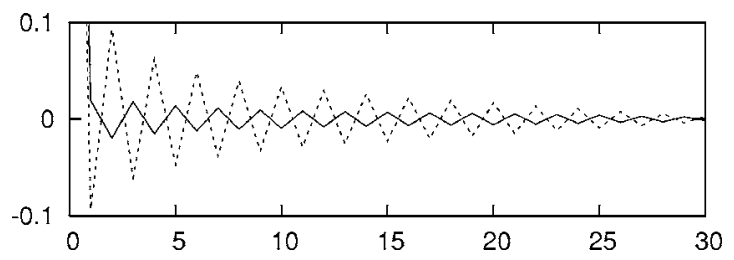

FIG. 5. Normalized correlation function $\nu_{i k}$ of Eq. (25) of waiting times calculated for the stochastic variable $\xi(t)$. Solid and dashed lines are for $H=1 / 3$ and $H=2 / 3$, respectively. Long-range correlations with alternating sign are clearly seen and should be compared to the case of Fig. 2, where the correlation function drops exactly to zero, after the first step. 
eration of the complex process are introduced through the correlation function of velocity-i.e., nonrenewal stochastic variable $\xi(t)$. The particle displacement $x(t)$ is obtained as a result of integration of the velocity, and it generates the renewal events at the moments when the particle crosses the origin $x=0$. At the same time the dispersion of the coordinate exhibits anomalous scaling $\propto t^{H}$ with $H \neq 1 / 2$. The model also establishes a simple relation, Eq. (3), between the scaling exponents $H$ and Lévy index $\theta$. According to this relation the ordinary diffusion $\theta=H=1 / 2$ is the only case when the displacement exhibits scaling $\propto \sqrt{t}$, without traces of cooperation. From this point of view the ordinary diffusion is a singularity since any deviation from the condition $\theta=1 / 2$ leads to cooperation, making the diffusion process anomalous. Unlike this singular case, a continuum of $\theta$ 's corresponds to anomalous diffusion along axis $x$, which, thus, should be considered as a regular case of the FBM.

The proposed RC dynamical model, in application to the BQD signal, is very promising since it extends the current approach, limited to the singular case $\theta=1 / 2$, to the whole range of $\theta$ 's. It also explains the relation between the renewal character of the signal and the expected cooperative properties of BQD's.

It is obvious that Eq. (1) is irrelevant to the proposed RC model, as well as to the RL FBM, since both these models are defined for $t>0$. However, in the scaling regime $\left|t_{1}-t_{2}\right|$ $\rightarrow \infty$ the RC model exhibits the asymptotical behavior of Eq. (23) — namely, the same properties as ordinary FBM. Equation (1), which is usually interpreted as a form of infinite memory, is related to Eq. (23), which is not a manifestation of memory, but rather is a consequence of the ergodicity breakdown [5]. These results will be discussed in a separate publication.

\section{ACKNOWLEDGMENTS}

We thankfully acknowledge the Welch Foundation and the ARO for support of this research through Grants Nos. B-1577 and W911NF-05-1-0059, respectively.
[1] M. Kuno, D. P. Fromm, H. F. Hamann, A. Gallagher, and D. J. Nesbitt, J. Chem. Phys. 115, 1028 (2001).

[2] F. Cichos, J. Martin, and C. von Borczyskowski, Phys. Rev. B 70, 115314 (2004).

[3] X. Brokmann, J.-P. Hermier, G. Messin, P. Desbiolles, J.-P. Bouchaud, and M. Dahan, Phys. Rev. Lett. 90, 120601 (2003).

[4] S. Bianco, P. Grigolini, and P. Paradisi, J. Chem. Phys. 123, 174704 (2005).

[5] G. Margolin and E. Barkai, Phys. Rev. Lett. 94, 080601 (2005).

[6] G. Aquino, L. Palatella, and P. Grigolini, Phys. Rev. Lett. 93, 050601 (2004).

[7] J. Tang and R. A. Marcus, Phys. Rev. Lett. 95, 107401 (2005).

[8] J. Klafter and I. M Sokolov, Phys. World 18, 29 (2005).

[9] B. B. Mandelbrot, The Fractal Geometry of Nature (Freeman, New York, 1977); J. Feder, Fractals (Plenum Press, New York, 1988).

[10] S. C. Lim and S. V. Muniandy, Phys. Rev. E 66, 021114 (2002).

[11] E. Barkai, Phys. Rev. Lett. 90, 104101 (2003).

[12] R. Failla, P. Grigolini, M. Ignaccolo, and A. Schwettmann, Phys. Rev. E 70, 010101(R) (2004).

[13] E. Barkai and Y.-C. Cheng, J. Chem. Phys. 118, 6167 (2003).

[14] R. Metzler and J. Klafter, Phys. Rep. 339, 1 (2000).

[15] W. Feller, An Introduction to Probability Theory and Its Applications (Wiley, New York, 1968, 1971), Vols. 1 and 2.

[16] M. Ding and W. Yang, Phys. Rev. E 52, 207 (1995).

[17] C. Godréche and J. M. Luck, J. Stat. Phys. 104, 314 (2001).
[18] J. Krug, H. Kallabis, S. N. Majumdar, S. J. Cornell, A. J. Bray, and C. Sire, Phys. Rev. E 56, 2702 (1997).

[19] J. Merikoski, J. Maunuksela, M. Myllys, J. Timonen, and M. J. Alava, Phys. Rev. Lett. 90, 024501 (2003).

[20] S. N. Majumdar and D. Das, Phys. Rev. E 71, 036129 (2005).

[21] J. Krug and H. T. Dobbs, Phys. Rev. Lett. 76, 4096 (1996).

[22] G. Margolin and E. Barkai, J. Chem. Phys. 121, 1566 (2004).

[23] U. Weiss, Quantum Dissipative Systems (World Scientific, Singapore, 1999).

[24] N. Pottier, Physica A 317, 371 (2003).

[25] We use $\Phi_{\xi}(\tau)=\left[15 \exp (-\tau / 2)-(1+\tau / 10)^{-4 / 3}\right] / 14$ for $\delta=4 / 3$ and $\Phi_{\xi}(\tau)=(1+\tau)^{-2 / 3}$ for $\delta=2 / 3$ in the numerical calculations. The tails of these correlation functions at $t \rightarrow \infty$ follow the asymptotic behavior required by Eq. (12), and also the compensation condition (13) is satisfied for $\delta>4 / 3$.

[26] M. Annunziato, P. Grigolini, and J. Riccardi, Phys. Rev. E 61, 4801 (2000).

[27] S. V. Lim and V. M. Sithi, Phys. Lett. A 206, 311 (1995).

[28] U. Kuhl, F. M. Izrailev, A. A. Krokhin, and H.-J. Stöckmann, Appl. Phys. Lett. 77, 633 (2000); F. M. Izrailev, A. A. Krokhin, and S. E. Ulloa, Phys. Rev. B 63, 041102(R) (2001).

[29] G. Godréche and J. Luck, J. Stat. Phys. 104, 489 (2001).

[30] G. Aquino, M. Bologna, P. Grigolini, and B. J. West, Phys. Rev. E 70, 036105 (2004).

[31] For $t_{a}>0$ the weight of short sojourn times is suppressed and it is enhanced for large times. Therefore, the waiting time distributions for different $t_{a}$ cross each other. On the contrary, the maximum of survival probability is shifted towards the origin. 